\title{
A inserção e a distribuição dos Terapeutas Ocupacionais no Sistema Único de Saúde do Estado de Minas Gerais entre os anos de 2005 e 2015
}

\section{The inclusion and distribution of Occupational Therapists in the Brazilian Unified Health System in Minas Gerais between the years 2005 and 2015}

\author{
Bruno Souza Bechara Maxta ${ }^{1}$, Alessandro Rodrigo Pedroso Tomasi ${ }^{2}$, \\ Melina Alves de Camargos ${ }^{3}$
}

http://dx.doi.org/10.11606/issn.2238-6149.v28i2p147-155

\begin{abstract}
Bechara Maxta BS, Tomasi ARP, Camargos MA. A inserção e a distribuição dos Terapeutas Ocupacionais no Sistema Único de Saúde do Estado de Minas Gerais entre os anos de 2005 e 2015. Rev Ter Ocup Univ São Paulo. 2017 maio-ago.;28(2):147-55.
\end{abstract}

RESUMO: Introdução: Estudos sobre os terapeutas ocupacionais no Sistema Único de Saúde (SUS) não abordam a inserção e a distribuição do profissional como importantes conhecimentos para a educação e planejamento assistencial. Objetivo: Identificar a inserção e a distribuição dos terapeutas ocupacionais atuantes no SUS do estado de Minas Gerais entre os anos de 2005 e 2015. Procedimentos Metodológicos: Pesquisa documental de caráter descritivo com documentos do Cadastro Nacional de Estabelecimentos de Saúde. Ferramentas da estatística descritiva foram utilizadas para a análise dos dados das variáveis, a saber: profissionais atuantes, macrorregiões de saúde, população e ano. Resultados: O número de terapeutas ocupacionais atuantes no SUS apresentou variação de crescimento e descentralização municipal em todas as macrorregiões de saúde. Conclusões: A inserção e da distribuição profissional nos equipamentos de saúde são informações necessárias para identificação da presença e planejamento da participação e formação do profissional no SUS.

DESCRITORES: Sistema único de saúde; Terapia ocupacional; Área de atuação profissional; Brasil/etnologia.
Bechara Maxta BS, Tomasi ARP, Camargos MA. The inclusion and distribution of Occupational Therapists in the Brazilian Unified Health System in Minas Gerais between the years 2005 and 2015. Rev Ter Ocup Univ São Paulo. 2017 May-Aug.;28(2):147-55.

ABSTRACT: Introduction: Studies on the occupational therapists in the Brazilian Unified Health System (SUS) do not focus on the insertion and the distribution of professionals as important knowledge for education and care planning. Objective: To identify the insertion and distribution of occupational therapists working in SUS in the state of Minas Gerais between 2005 and 2015. Methodological Procedures: Descriptive documentary research with documents from the National Registry of Health Establishments. Descriptive statistics tools were used for the data analysis considering the variables: working professionals, macro-regions of health, number of population and year. Results: The number of occupational therapists working in the SUS presented a variation of growth and municipal decentralization in all health macro-regions. Conclusions: The insertion and the professional distribution in the health equipment are important information to identify the presence and to plan the participation and the formation of the professional for the SUS.

KEYWORDS: Unified health system; Occupational therapy; Professional practice location; Brazil/ethnology.

Trabalho é parte integrante do projeto de pesquisa "Terapeutas Ocupacionais no Sistema Único de Saúde em Minas Gerais" desenvolvido pelo Departamento de Terapia Ocupacional da Universidade Federal de Minas Gerais.

1. Departamento de Terapia Ocupacional, Universidade Federal de Minas Gerais - UFMG, Belo Horizonte, MG, Brasil. E-mail: brunobechara@ufmg.br

2. Departamento de Terapia Ocupacional, Universidade Federal de Minas Gerais - UFMG, Belo Horizonte, MG, Brasil. E-mail: arp.tomasi@gmail.com

3. Programa de Residência Multiprofissional em Saúde Coletiva e Atenção Primária Departamento de Medicina Preventiva da FMUSP, São Paulo, SP, Brasil; melinacamargos.to@gmail.com

Autor para correspondência: Bruno Souza Bechara Maxta. Escola de Educação Física, Fisioterapia e Terapia Ocupacional, Universidade Federal de Minas Gerais. Av. Pres. Antônio Carlos, 6627. Campus Pampulha, Belo Horizonte, MG, Brasil. CEP: 31270-901. E-mail: brunobechara@ufmg.br 


\section{INTRODUÇÃO}

$\mathrm{O}$ Sistema Único de Saúde (SUS) representa o conjunto articulado de ações e de serviços públicos direcionados às necessidades de saúde da população brasileira. O planejamento e a organização da assistência à saúde são desenvolvidos sob a perspectiva do pacto interfederativo em que a regionalização e a hierarquização de serviços de diferentes complexidades são importantes diretrizes para a organização das Redes de Atenção à Saúde (RAS) ${ }^{1}$.

A regionalização em saúde se configura como uma diretriz organizacional do SUS para a descentralização das suas ações entre municípios pertencentes a uma determinada região de saúde. Neste processo, o princípio da hierarquização colabora para a definição dos serviços e ações que serão priorizados por cada esfera de governo, segundo o grau de complexidade tecnológica, pactuações e corresponsabilidades na composição das RAS².

As RAS compõem conjuntos de serviços de saúde, vinculados entre si por objetivos comuns e por ações cooperativas e interdependentes em uma determinada região de saúde. O planejamento, a organização e a gestão colegiada das ofertas de saúde buscam integrar os serviços da Atenção Primária em Saúde (APS), da Vigilância em Saúde, da Assistência Ambulatorial e Hospitalar de média e alta complexidades loco-regionais ${ }^{3}$.

Neste cenário, os profissionais de saúde se destacam como protagonistas para a tessitura das RAS loco-regionais, particularmente no desenvolvimento/ implementação de tecnologias de gestão e de assistência que respondam às necessidades de saúde territoriais e de organização do SUS. É indicado ao terapeuta ocupacional a sua contribuição para a formulação, implementação e controle social das políticas e das ações de saúde em seus lugares de intervenção ${ }^{4}$. No SUS, são lugares potenciais de atuação do terapeuta ocupacional, o cotidiano dos serviços de atenção e vigilância em saúde no contexto da APS, o campo da saúde mental, da reabilitação física e da saúde da pessoa com deficiência ${ }^{5}$.

Estudos sobre a inserção e o envolvimento do terapeuta ocupacional no SUS são pouco direcionados às discussões sobre a presença, distribuição, planejamento assistencial e formação profissional. Sob o desafio de iniciar a compreensão do processo de inserção, distribuição, mobilidade e participação do terapeuta ocupacional no SUS do estado de Minas Gerais, o presente estudo teve como objetivo identificar a inserção e a distribuição dos terapeutas ocupacionais atuantes no SUS deste estado entre os anos de 2005 e 2015.

\section{PROCEDIMENTOS METODOLÓGICOS}

Tratou-se de um estudo descritivo, realizado por meio de pesquisa documental. A pesquisa documental se caracteriza pelo estudo de fontes de dados primárias e/ ou secundárias sobre determinados assuntos nos quais o pesquisador busca, de forma organizada e retrospectiva, elementos que possam contribuir para explicar uma determinada realidade ${ }^{6}$.

Os dados documentais utilizados foram coletados na base de dados do Cadastro Nacional de Estabelecimentos de Saúde (CNES) através do site do Departamento de Informática do Sistema Único de Saúde (DATASUS) no mês de março de 2015.

O CNES é uma base de dados de acesso público, de atualização semanal, que disponibiliza informações de saúde das esferas municipais, estaduais e federal nos âmbitos público e privados ${ }^{7}$. Ao DATASUS compete a gestão da informação e de ações de informatização no âmbito do SUS, no sentido de apoiar os estados e municípios no gerenciamento de bancos de dados sobre saúde ${ }^{8}$.

Os dados coletados foram compostos pelo quantitativo absoluto de terapeutas ocupacionais inseridos nos diferentes estabelecimentos de saúde da administração direta, indireta e privada que atendem ao SUS nos municípios do estado de Minas Gerais entre o período de agosto de 2005 e janeiro de 2015.

Para o estudo em questão, foram utilizados os seguintes menus no sítio eletrônico do DATASUS, na sequência indicada: Informações de Saúde (TABNET); Rede Assistencial; CNES/Recursos Humanos até julho de 2007 - Ocupações classificadas pela CBO 1994 e CNES/Recursos Humanos a partir de agosto de 2007 Ocupações classificadas pela CBO 2002. A utilização dos dois últimos menus é justificada pelo período selecionado no estudo. Na sequência, foi selecionada a opção Ocupações, determinada a abrangência geográfica (com seleção por mapa ou lista) sendo escolhida a opção Minas Gerais. Os dados selecionados corresponderam à correlação entre as opções Linha 'Município', Coluna 'Tipo de Estabelecimento', Conteúdo 'Atende o SUS' e ocupação 'Terapeuta Ocupacional'. Neste processo, foram marcadas as opções de exibição de linhas zeradas, para que os municípios que não contivessem terapeutas ocupacionais aparecessem na busca. 
Os dados foram organizados em tabelas, geradas pelo sítio eletrônico do DATASUS, armazenados em planilhas eletrônicas e arquivados em pastas virtuais individualizadas. Para a leitura das tabelas, foi utilizado software Open Office. A utilização deste programa foi necessária para a edição de dados e leitura destes pelo aplicativo de geoprocessamento utilizado no processamento e organização das informações. Os mapas foram construídos no software QuantumGIS (versão 2.2). Os achados de pesquisa foram dispostos em mapas, tabelas e gráficos, para posterior análise.

A análise dos dados se deu de forma descritiva, a partir do material produzido, com observância da apresentação e distribuição espacial de terapeutas ocupacionais nos municípios do estado e; análise estatística descritiva das tabelas, com utilização dos parâmetros frequência absoluta, frequência relativa, variação (considerando os valores iniciais diferentes de zero e finais) e a média geral do período, considerando as regiões de saúde de Minas Gerais.

\section{RESULTADOS}

No estado de Minas Gerais, a regionalização do SUS está organizada em 13 Macrorregiões (MRS) e que incorporam 853 municípios, em uma área de 580.720,8 $\mathrm{km}^{2}$ e população estimada em 2005 de 19.237 .450 e em 2015 de 20.734.097 habitantes ${ }^{9}$.
No ano de 2005, foram identificados 566 terapeutas ocupacionais atuantes no SUS distribuídos nas 13 MRS do estado, independente da natureza administrativa, do serviço em que foram vinculados e/ou das formas de contratualização para o trabalho em saúde. Em 2015, este quantitativo foi de 1269 (variação de 124\%). Neste conjunto, todas as MRS apresentaram variações de crescimento do número absoluto de terapeutas ocupacionais. Os maiores crescimentos proporcionais foram identificados nas MRS Jequitinhonha (1700\%), MRS Nordeste (280\%), MRS Leste (253\%) e MRS Centro Sul (245\%). As MRS Centro ( $\mathrm{X}=474$, onde $\mathrm{X}$ é o número de terapeutas ocupacionais na MRS) e MRS Jequitinhonha $(X=8)$ apresentaram a maior e a menor médias profissionais. Estas informações são apresentadas na Tabela 1 e ilustradas no Mapa (Figura 1).

Em 2005, 140 municípios registraram a presença do terapeuta ocupacional em suas RASs. Destes, 87,14\% apresentaram de um a cinco profissionais em seus quadros de trabalhadores. No ano de 2015, o número de municípios que integraram terapeuta ocupacional aumentou 16,64\%. Naquele ano, 69,51\% dos municípios não apresentaram o terapeuta ocupacional atuante no SUS em suas redes assistenciais; $25,32 \%$ dos municípios se enquadraram no gradiente de um a cinco profissionais; outros $4,33 \%$ apresentaram o gradiente de seis a 20 e $0,82 \%$ o gradiente de mais de 20 profissionais (Gráfico 1).

Tabela 1 - Frequência absoluta, frequência relativa, variação e média dos terapeutas ocupacionais atuantes no SUS entre os anos de 2005 e 2015

\begin{tabular}{|c|c|c|c|c|c|c|c|c|c|c|c|c|c|c|c|c|c|c|c|c|c|c|c|c|}
\hline \multirow{2}{*}{$\begin{array}{l}\text { Macrorregiões } \\
\text { de Saúde }\end{array}$} & \multicolumn{2}{|c|}{2005} & \multicolumn{2}{|c|}{2006} & \multicolumn{2}{|c|}{2007} & \multicolumn{2}{|c|}{2008} & \multicolumn{2}{|c|}{2009} & \multicolumn{2}{|c|}{2010} & \multicolumn{2}{|c|}{2011} & \multicolumn{2}{|c|}{2012} & \multicolumn{2}{|c|}{2013} & \multicolumn{2}{|c|}{2014} & \multicolumn{2}{|c|}{2015} & \multirow{2}{*}{\begin{tabular}{c|} 
Variação \\
$\%$ o
\end{tabular}} & \multirow{2}{*}{$\begin{array}{c}\text { Média } \\
\mathrm{X}\end{array}$} \\
\hline & fi & fp & fi & fp & fi & fp & fi & fp & fi & fp & fi & fp & fi & fp & fi & fp & fi & fp & fi & fp & fi & fp & & \\
\hline Centro & 290 & 51 & 299 & 51 & 309 & 48 & 399 & 51 & 496 & 52 & 522 & 51 & 528 & 48 & 550 & 49 & 563 & 48 & 614 & 49 & 646 & 51 & $123 \%$ & 474 \\
\hline Centro Sul & 20 & 4 & 18 & 3 & 30 & 5 & 42 & 5 & 53 & 6 & 71 & 7 & 74 & 7 & 65 & 6 & 70 & 6 & 73 & 6 & 69 & 5 & $245 \%$ & 53 \\
\hline Jequitinhonha & 1 & 0 & 1 & 0 & 1 & 0 & 3 & 0 & 6 & 1 & 7 & 1 & 7 & 1 & 11 & 1 & 11 & 1 & 17 & 1 & 18 & 1 & $1700 \%$ & 8 \\
\hline Leste & 15 & 3 & 18 & 3 & 18 & 3 & 20 & 3 & 31 & 3 & 27 & 3 & 32 & 3 & 43 & 4 & 44 & 4 & 49 & 4 & 53 & 4 & $253 \%$ & 32 \\
\hline Leste do Sul & 10 & 2 & 9 & 2 & \begin{tabular}{|l|}
11 \\
\end{tabular} & 2 & 16 & 2 & 15 & 2 & 18 & 2 & 24 & 2 & 22 & 2 & 25 & 2 & 27 & 2 & 26 & 2 & $160 \%$ & 18 \\
\hline Nordeste & 5 & 1 & 6 & 1 & 8 & 1 & 9 & 1 & 9 & 1 & 13 & 1 & 13 & 1 & 12 & 1 & 15 & 1 & 17 & 1 & 19 & 1 & $280 \%$ & 11 \\
\hline Noroeste & 20 & 4 & 18 & 3 & 18 & 3 & 20 & 3 & 22 & 2 & 19 & 2 & 23 & 2 & 24 & 2 & 26 & 2 & 28 & 2 & 26 & 2 & $30 \%$ & 22 \\
\hline Norte & 20 & 4 & 19 & 3 & 17 & 3 & 20 & 3 & 28 & 3 & 31 & 3 & 32 & 3 & 31 & 3 & 29 & 2 & 29 & 2 & 29 & 2 & $45 \%$ & 26 \\
\hline Oeste & 34 & 6 & 33 & 6 & 39 & 6 & 38 & 5 & 48 & 5 & 50 & 5 & 51 & 5 & 53 & 5 & 51 & 4 & 51 & 4 & 55 & 4 & $62 \%$ & 46 \\
\hline Sudeste & 22 & 4 & 29 & 5 & 33 & 5 & 36 & 5 & 45 & 5 & 47 & 5 & 64 & 6 & 65 & 6 & 66 & 6 & 62 & 5 & 64 & 5 & $191 \%$ & 48 \\
\hline Sul & 75 & 13 & \begin{tabular}{|l|}
79 \\
\end{tabular} & 13 & 92 & 14 & 100 & 13 & 119 & 12 & 133 & 13 & 149 & 14 & 155 & 14 & 165 & 14 & 172 & 14 & 156 & 12 & $108 \%$ & 127 \\
\hline $\begin{array}{l}\text { Triângulo } \\
\text { do Norte }\end{array}$ & 26 & 5 & 26 & 4 & 30 & 5 & 35 & 5 & 37 & 4 & 40 & 4 & 41 & 4 & 41 & 4 & 44 & 4 & 47 & 4 & 44 & 3 & $69 \%$ & 37 \\
\hline $\begin{array}{l}\text { Triângulo } \\
\text { do Sul }\end{array}$ & 28 & 5 & 31 & 5 & 39 & 6 & 40 & 5 & 46 & 5 & 51 & 5 & 52 & 5 & 56 & 5 & 62 & 5 & 61 & 5 & 64 & 5 & $129 \%$ & 48 \\
\hline Total & 566 & 100 & 586 & 100 & \begin{tabular}{|l|}
645 \\
\end{tabular} & 100 & 778 & 100 & 955 & 100 & 1029 & 100 & 1090 & 100 & 1128 & 100 & 1171 & 100 & \begin{tabular}{|l|}
1247 \\
\end{tabular} & 100 & \begin{tabular}{l|l|}
1269 \\
\end{tabular} & 100 & $124 \%$ & 951 \\
\hline
\end{tabular}

Fonte: Os autores 



Fonte: Os autores.

Figura 1 - Mapa, representação quantitativa dos terapeutas ocupacionais atuantes no SUS nos municípios do estado de Minas Gerais nos anos de 2005 e 2015 
Gráfico 1 - Relação entre o número de municípios e o número de terapeutas ocupacionais atuantes no SUS do estado de Minas Gerais entre os anos de 2005 e 2015



Fonte: Os autores.

Entre os anos de 2005 e 2015, o número de terapeutas ocupacionais atuantes no SUS por município cresceu de 0,66 para 1,48. Os municípios com menos de 10.000 habitantes e municípios entre 10.001 e 50.000 habitantes apresentaram os maiores crescimentos absolutos de profissionais, com os valores $527 \%$ e $161 \%$, respectivamente (Gráfico 2). Do mesmo modo, o número de terapeutas ocupacionais por habitante aumentou de 2,94e-5 para 6,12e-5. Foi possível identificar esta relação em todas as MRS, à exceção da MR Noroeste. $\mathrm{Na}$ prática, isso significa que a cobertura profissional por terapeutas ocupacionais atendendo nos serviços do SUS dobrou no período. As MRS com as maiores e menores coberturas assistenciais por terapeutas ocupacionais foram identificados nas MRS Centro (9,96e-5) e MRS Norte (1,74e-5) (Tabela 2).

Gráfico 2 - Relação entre o porte populacional municipal e o número de terapeutas ocupacionais atuantes no SUS do estado de Minas Gerais entre os anos de 2005 e 2015

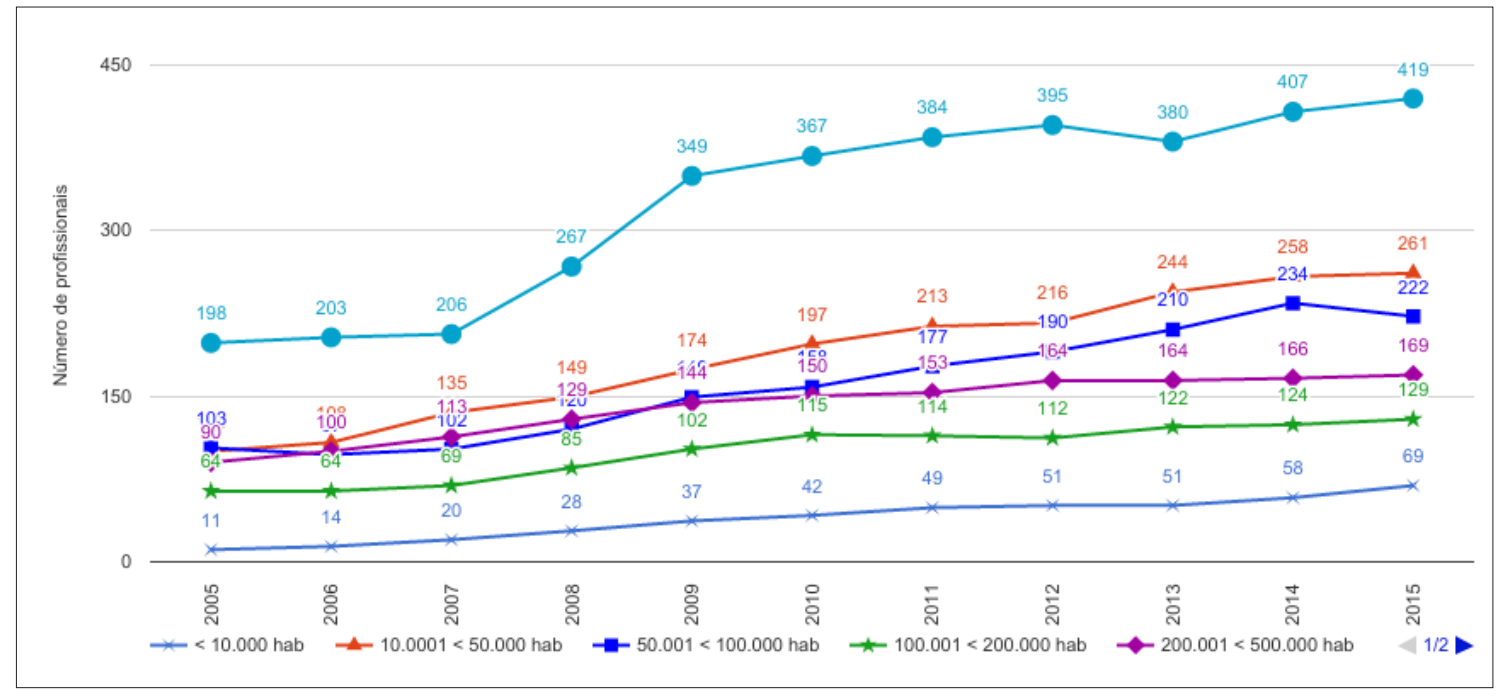

Fonte: Os autores. 
Bechara Maxta BS, et al. A inserção e a distribuição dos Terapeutas Ocupacionais. Rev Ter Ocup Univ São Paulo. 2017 maio/ago.;28(2):147-55.

Tabela 2 - Relação do número absoluto de terapeutas ocupacionais por habitantes nas MRS em 2005 e 2015

\begin{tabular}{l|c|c|c|c|c|c|c}
\hline \multirow{2}{*}{ Macrorregião de Saúde } & \multirow{2}{*}{$\begin{array}{c}\text { Municípios } \\
\text { (n) }\end{array}$} & \multicolumn{2}{|c|}{ População (hab.) } & \multicolumn{2}{c|}{ Profissionais (n) } & \multicolumn{2}{c}{ Relação hab./ profissionais } \\
\cline { 3 - 7 } & 103 & $\mathbf{2 0 0 5}$ & $\mathbf{2 0 1 5}$ & $\mathbf{2 0 0 5}$ & $\mathbf{2 0 1 5}$ & $\mathbf{2 0 0 5}$ & $\mathbf{2 0 1 5}$ \\
\hline Centro & 51 & 724.410 & 780.011 & 20 & 646 & $20.914,10$ & $10.031,20$ \\
\hline Centro Sul & 28 & 282.569 & 296.870 & 1 & 18 & 282.569 & 16.493 \\
\hline Jequitinhonha & 86 & 1.415 .997 & 1.523 .095 & 15 & 53 & $94.399,80$ & 28.738 \\
\hline Leste & 53 & 660.054 & 694.964 & 10 & 26 & $66.055,40$ & $26.729,40$ \\
\hline Leste do Sul & 58 & 881.648 & 931.946 & 5 & 19 & $176.329,60$ & $49.049,80$ \\
\hline Nordeste & 33 & 637.784 & 931.946 & 20 & 26 & $31.889,20$ & 35.844 \\
\hline Noroeste & 86 & 1.544 .294 & 1.661 .130 & 20 & 29 & $77.214,70$ & $57.280,30$ \\
\hline Norte & 54 & 1.114 .914 & 1.244 .944 & 34 & 55 & $32.791,60$ & $22.635,30$ \\
\hline Oeste & 94 & 1.541 .964 & 1.651 .433 & 22 & 64 & $70.089,30$ & $25.803,60$ \\
\hline Sudeste & 153 & 2.594 .480 & 2.755 .109 & 75 & 156 & $34.593,10$ & 17.661 \\
\hline Sul & 27 & 1.137 .296 & 1.260 .398 & 26 & 44 & $43.742,10$ & $28.645,40$ \\
\hline Triângulo do Norte & 27 & 636.940 & 752.948 & 28 & 64 & $22.747,80$ & $11.764,80$ \\
\hline Triângulo do Sul & $\mathbf{8 5 3}$ & $\mathbf{1 9 . 2 3 7 . 4 5 0}$ & $\mathbf{2 0 . 7 3 4 . 0 9 7}$ & $\mathbf{5 6 6}$ & $\mathbf{1 2 6 9}$ & $\mathbf{3 3 . 9 8 8 , 4 0}$ & $\mathbf{1 6 . 3 3 8 , 9 0}$ \\
\hline Total & \multicolumn{3}{|c|}{} & & & &
\end{tabular}

Fonte: Os autores.

\section{DISCUSSÃO}

Os valores encontrados indicaram a ocorrência do fenômeno de crescimento do número absoluto de terapeutas ocupacionais com concentração nos municípios das regiões de saúde metropolitanas e a tendência de sua distribuição para os municípios de menor porte habitacional no estado.

Este fenômeno pode ser justificado pelos investimentos municipal e estadual oriundos do conjunto de políticas públicas de educação e de saúde, vigentes entre 2005 e 2015, as quais impulsionaram, por um lado, as instituições de ensino superior, para a redefinição de seus projetos pedagógicos voltados para a formação profissional para o SUS e, por outro, os municípios a investirem na contratação do profissional, pela administração direta e/ou complementar a este sistema.

Em 2002, por definição do Conselho Nacional de Educação do Ministério da Educação, os cursos de graduação da área da saúde das Instituições de Ensino Superior (IES) passaram a adequar os projetos pedagógicos a partir das novas Diretrizes Curriculares Nacionais do Curso de Graduação. A resolução específica para os cursos de graduação em terapia ocupacional reorientou os eixos de formação deste profissional a partir de competências e habilidades profissionais para responder às necessidades e as lacunas do SUS ${ }^{10}$. Ao longo do período estudado, seis cursos de graduação ofereceram oportunidades de formação profissional para o SUS ou para a sua complementaridade ${ }^{11}$. Destes, quatro cursos de graduação (75\% IESs) operaram na MRS Centro - MRS que se manteve com a maior concentração profissional do período estudado (51\%).

A Norma Operacional de Assistência à Saúde (NOAS), publicada em 2002, ampliou as responsabilidades dos municípios para a organização e oferta de serviços de APS, bem como definiu a regionalização como estratégia central para estes organizarem os demais serviços oferecidos à população ${ }^{12}$. A importância dos municípios desenvolverem diagnósticos sobre a situação da força de trabalho em saúde, quer seja pela avaliação da sua quantidade, composição, qualidade e definição do quadro necessário para o funcionamento do sistema, incluindo a proposta contratação de pessoal, entre outras atividades inerentes ao tema, visando o planejamento para a execução da gestão e das ações e serviços de saúde, pelos diferentes níveis de gestão do SUS, foram apontadas com a publicação dos princípios e diretrizes para a Norma Operacional Básica de Recursos Humanos para o SUS ${ }^{13}$.

No intuito de apoiar a implementação das normas vigentes, o documento Pacto pela Saúde representou o compromisso interfederativo em responder aos principais desafios identificados no SUS. Este documento apontou para a necessidade dos municípios implementarem um conjunto de reformas institucionais voltadas à responder a eficiência, a qualidade e as responsabilidades sobre a assistência às necessidades loco-regionais do sistema, considerando os eixos da APS, Média e Alta Complexidade da Assistência, da Vigilância em Saúde, da Assistência Farmacêutica e da Gestão do SUS ${ }^{14}$. 
Neste compromisso, o Pacto pela Vida e de Gestão representaram acordos interfederativos específicos para a gestão e a assistência do SUS, centralizando investimentos na APS, partir de áreas estratégicas e parâmetros propostos pelas áreas técnicas do Ministério da Saúde e consensuados nos colegiados gestores regionais. Entre as metas estabelecidas por este documento, destacaram-se o aprimoramento da inserção de profissionais nas redes locais de saúde, por meio de vínculos de trabalho que favorecessem o provimento e a fixação dos mesmos na APS, e a contratualização de prestadores de serviços complementares de média e alta complexidade acompanhados pelas comissões intergestoras regionais e da secretaria estadual de saúde ${ }^{14}$.

Desde então, um conjunto de portarias ministeriais foram publicadas de forma a estimular a ampliação e a qualificação da organização do SUS sob a perspectiva da integralidade em RAS temáticas ${ }^{15}$, a saber: para o cuidado à gestante e à criança ${ }^{16,17}$; às situações de urgências e emergências ${ }^{18}$; às necessidades de saúde mental e decorrentes ao uso de drogas ${ }^{19}$; à pessoa em situação de doenças ou condições de crônicas de saúde ${ }^{20}$ incluindo a deficiência ${ }^{21}$.

Nestas RAS, o terapeuta ocupacional vem sendo apresentado como um dos profissionais que podem compor as equipes de saúde nos serviços assistenciais da APS; da urgência e emergência; da atenção psicossocial; da atenção ambulatorial especializada e hospitalar do SUS. $\mathrm{Na}$ Rede de Atenção Psicossocial, o terapeuta ocupacional é indicado para a composição das equipes de referência dos serviços de atenção psicossocial, das unidades de acolhimento e do consultório na rua. $\mathrm{Na}$ Rede de Cuidados à Pessoa com Deficiência, este profissional é indicado para a composição das equipes multiprofissionais em diferentes serviços ambulatoriais e hospitalares que respondem a assistência. Na Rede de Urgência e Emergência, o terapeuta ocupacional é indicado para compor as equipes de apoio nos serviços da Atenção Domiciliar. No contexto da APS, o terapeuta ocupacional é apresentado como profissional do Núcleo de Apoio à Saúde da Família e da Academia da Saúde ${ }^{22}$.

Neste processo, a Secretaria Estadual de Saúde de Minas Gerais buscou apoiar técnica e financeiramente os municípios do estado para a garantia da prestação dos serviços de saúde à sua população. O Plano Plurianual de Ação Governamental do quadriênio 2004-2007³ e os Planos Estaduais de Saúde dos quadriênios 2008$2011^{24}$ e 2012-2015²5, ao considerarem o Plano Diretor de Regionalização da Saúde de Minas Gerais (PDR/MG) de 2002 (atualizado em 2011), o Pacto pela Saúde, e as análises da situação de saúde no estado, indicaram uma série de estratégias para o enfrentamento dos problemas de saúde apresentados pelos municípios.

Destas estratégias, o projeto Saúde em Casa buscou garantir a universalização do acesso à saúde por meio a ampliação de serviços na APS dos municípios de pequeno e médio porte. O projeto Viva a Vida representou o empenho para a redução da mortalidade materno-infantil. Projetos adicionais buscaram impulsionar a Regionalização da rede de Urgência e Emergência no estado.

Este cenário possibilitou, portanto, aos atores do SUS a reconfigurarem as redes assistenciais municipais e regionais, aproveitando os recentes investimentos oriundos, em grande parte, das portarias ministeriais das RAS temáticas direcionadas para a APS, para a Saúde Mental e para a Urgência e Emergência. As portarias ministeriais orientaram e apoiaram diretamente os municípios na implementação e gestão de novos serviços, pela inserção de novos profissionais, bem como orientou os estados a se corresponsabilizarem pela organização regional de serviços ambulatoriais e hospitalares de média e alta complexidade. A ampliação do número absoluto dos municípios que inseriram terapeuta ocupacional em suas redes assistenciais e a aumento da relação terapeuta ocupacional por habitante no estado, refletem tais investimentos.

\section{CONCLUSÕES}

O profissional terapeuta ocupacional foi inserido, gradativamente, nas RAS das MRS do estado de Minas Gerais, apoiados por políticas públicas federais e estaduais, os quais ampliaram e os serviços de saúde em suas redes loco-regionais. Neste sentido, o número de municípios com nenhum terapeuta ocupacional diminuiu sistematicamente no período, em concomitância com o aumento da relação terapeuta ocupacional por habitante. Ainda, observou-se um processo de interiorização do profissional.

Apesar do aumento do número de terapeutas ocupacionais e da tendência da descentralização destes profissionais dos grandes centros urbanos para cidades de médio e pequeno porte do estado ao longo do período estudado, não se pode afirmar que isso significa a garantia do acesso da população aos serviços deste profissional.

Sobre estes aspectos, é convidativo aos profissionais, representantes de categoria profissional e acadêmicos trabalharem para a apresentação e inserção do profissional terapeuta ocupacional no estado. A luta pela ampliação do número de terapeutas ocupacionais que compõem os quadros de pessoal das prefeituras 
municipais, o estímulo à contratação de profissionais pela administração direta da saúde, a descentralização dos centros de formação e qualificação profissional são exemplos de ações que podem provocar a inserção dos terapeutas ocupacionais em municípios que ainda não contam com o trabalho deste profissional.

Neste movimento, a presença do profissional nos serviços de saúde e a sua participação nas arenas de planejamento, de gestão e de controle social no SUS de seu município tendem a ser uma importante forma de construir a inserção da terapia ocupacional em novos e outros serviços de saúde locais.

\section{REFERÊNCIAS}

1. Brasil. Presidência da República. Casa Civil. Subchefia para Assuntos Jurídicos. Lei n. 8.080 de 12 de setembro de 1990. Lei no 8.080, de 19 de Setembro de 1990. Dispõe sobre as condições para a promoção, proteção e recuperação da saúde, a organização e o funcionamento dos serviços correspondentes e dá outras providências. Diário Oficial da União, Brasília; 1990. Disponível em: https://www.planalto. gov.br/ccivil_03/leis/L8080.htm.

2. Mendes EV. As redes de atenção à saúde. . 2a ed. Brasília: Organização Pan-Americana de Saúde; 2011. Disponível em: http://apsredes.org/site2012/wp-content/uploads/2012/03/ Redes-de-Atencao-mendes2.pdf.

3. Malachias I, Leles FAG, Pinto MAS. Plano diretor de regionalização de saúde de Minas Gerais. Belo Horizonte: Secretaria de Estado de Saúde de Minas Gerais; 2010. Disponível em: http://www.saude.mg.gov.br/images/ documentos/Livro\%20Plano\%20Diretor\%20de\%20 Regionalizacao\%20-\%20ultima\%20versao.pdf.

4. Malfitano APS, Ferreira AP. Saúde pública e terapia ocupacional: apontamentos sobre relações históricas e atuais. Rev de Ter Ocup Univ São Paulo. 2011;22(2):102-9. DOI: http://dx.doi.org/10.11606/issn.2238-6149.v22i2p102-109.

5. Beirão ROS, Alves CKA. Terapia ocupacional no SUS: refletindo sobre a normatização vigente. Cad Ter Ocup UFSCar. 2010;18(3):231-46. Disponível em: http://www. cadernosdeterapiaocupacional.ufscar.br/index.php/cadernos/ article/view/378/293.

6. Gil AC. Como elaborar projetos de pesquisa. São Paulo: Atlas; 2007.

7. Faveret ACSC. Prontuários de bases de dados: informação sistematizada para as contas de saúde no Brasil. Brasília: Ipea; 2009.
O estudo sobre a inserção e distribuição dos terapeutas ocupacionais nas MRS do estado de Minas Gerais foi o primeiro passo para o (re)conhecimento desta profissão frente aos desafios colocados na agenda da saúde do estado. Outros estudos sobre estes objetos, bem como sobre a natureza administrativa dos vínculos profissionais, a atuação profissional nos diferentes níveis de atenção à saúde do estado, ou mesmo sobre o processo de trabalho do terapeuta ocupacional nos equipamentos de saúde são necessários para melhor compreendermos e somarmos esforços para ampliarmos e transformarmos o trabalho do terapeuta ocupacional no SUS de Minas Gerais.

8. Brasil. Ministério da Saúde. Departamento de Informática do Sistema Único de Saúde. Histórico/Apresentação. Brasília: Secretaria Executiva MS;2016 [Acesso 21 dez. 2016]. Disponível em: http://w3.datasus.gov.br/datasus/datasus. php? area=363A6B0C0D0E0F363G6HIJd6L7M0N\&V Include $=.$. /site/texto.php.

9. Brasil. Ministério da Saúde. Departamento de Informática do Sistema Único de Saúde: População estimada por ano segundo município. Brasília; 2016 [Acesso 21 dez. 2016\}. Disponível em: http://datasus.gov.br.

10. Brasil. Ministério da Educação. Conselho Nacional de Educação. Câmara de Educação Superior. Resolução n. 6, de 19 de fevereiro de 2002. Institui Diretrizes Curriculares Nacionais do Curso de Graduação em Terapia Ocupacional. Diário Oficial da União, Brasília (DF); 2002. Disponível em: http://portal.mec.gov.br/cne/arquivos/pdf/CES062002.pdf.

11. Brasil. Ministério da Educação. Instituições de Educação Superior e Cursos Cadastrados. Brasília (DF); 2016. [Acesso 21 dez. 2016]. Disponível em: http://emec.mec.gov.br/.

12. Brasil. Ministério da Saúde. Secretaria de Assistência à Saúde. Departamento de Descentralização da Gestão da Assistência. Regionalização da assistência à saúde: aprofundando a descentralização com equidade no acesso - Norma Operacional da Assistência à Saúde: NOAS-SUS 01/02 e Portaria MS/GM n. ${ }^{\circ}$ 373, de 27 de fevereiro de 2002 e regulamentação complementar. Brasília (DF); 2002. Disponível em: http://siops.datasus.gov.br/Documentacao/ NOAS\%2001\%20de\%202002.pdf.

13. Brasil. Ministério da Saúde. Conselho Nacional de Secretários de Saúde. Princípios e diretrizes para a gestão do trabalho no SUS (NOB/RH-SUS). Brasília (DF); 2005. Disponível em: http://bvsms.saude.gov.br/bvs/publicacoes/NOB_RH_2005.pdf. 
14. Brasil. Ministério da Saúde. Secretaria Executiva. Departamento de Apoio à Descentralização. Regulamento dos pactos pela vida e de gestão. Brasília (DF); 2006. Disponível em: http://conselho.saude.gov.br/webpacto/regulacao.pdf.

15. Brasil. Ministério da Saúde. Portaria GM/MS n. 4.279, de 30 de dezembro de 2010. Estabelece diretrizes para a organização da Rede de Atenção à Saúde no âmbito do Sistema Único de Saúde (SUS). Diário Oficial da União, Brasília; 2010. Disponível em: http://conselho.saude.gov.br/ ultimas_noticias/2011/img/07_jan_portaria4279_301210.pdf.

16. Brasil. Ministério da Saúde. Secretaria de Atenção à Saúde. Departamento de Ações Programáticas Estratégicas. Política nacional de atenção integral à saúde da mulher. Brasília (DF); 2004. Disponível em: http://bvsms.saude.gov.br/bvs/ publicacoes/politica_nac_atencao_mulher.pdf.

17. Brasil. Ministério da Saúde. Portaria GM/MS n. 1.130 de 05 de agosto de 2015. Institui a Política Nacional de Atenção Integral à Saúde da Criança (PNAISC) no âmbito do Sistema Único de Saúde (SUS). Diário Oficial da União, Brasília; 2015. Disponível em: http://bvsms.saude.gov.br/bvs/ saudelegis/gm/2015/prt1130_05_08_2015.html.

18. Brasil. Ministério da Saúde. Portaria GM/MS n. 1.600 de 07 de julho de 2011. Reformula a Política Nacional de Atenção às Urgências e institui a Rede de Atenção às Urgências no Sistema Único de Saúde (SUS). Diário Oficial da União, Brasília; 2011. Disponível em: http://bvsms.saude.gov.br/ bvs/saudelegis/gm/2011/prt1600_07_07_2011.html.

19. Brasil. Ministério da Saúde. Portaria GM/MS n. 3.088 de 23 de dezembro de 2011. Institui a Rede de Atenção Psicossocial para pessoas com sofrimento ou transtorno mental e com necessidades decorrentes do uso de crack, álcool e outras drogas, no âmbito do Sistema Único de Saúde. Diário Oficial da União, Brasília; 2011. Disponível em: http://bvsms.saude. gov.br/bvs/saudelegis/gm/2011/prt3088_23_12_2011_rep.html.
20. Brasil. Ministério da Saúde. Portaria GM/MS n. 483 de 1 de abril de 2014. Redefine a Rede de Atenção à Saúde das Pessoas com Doenças Crônicas no âmbito do Sistema Único de Saúde (SUS) e estabelece diretrizes para a organização das suas linhas de cuidado. Diário Oficial da União, Brasília; 2014. Disponível em: http://bvsms.saude.gov.br/bvs/ saudelegis/gm/2014/prt0483_01_04_2014.html.

21. Brasil. Ministério da Saúde. Portaria GM/MS n. 793 de 24 de agosto de 2012. Institui a Rede de Cuidados à Pessoa com Deficiência no âmbito do Sistema Único de Saúde. Diário Oficial da União, Brasília; 2012. Disponível em: http://bvsms.saude.gov. br/bvs/saudelegis/gm/2012/prt0793_24_04_2012.html.

22. Camargos MA, Ferreira MVB, Maxta BSB, Tomasi ARP. A terapia ocupacional nas redes de atenção do Sistema Único de Saúde. In: Jornada Acadêmica de Terapia Ocupacional; Belo Horizonte, MG, 03-04 out. 2014. Disponível em: http://media. wix.com/ugd/810b1e_77f027d607ae4390b8eeffe497c2bc9f.pdf.

23. Minas Gerais. Secretaria de Estado de Planejamento e Gestão. Subsecretaria de Planejamento e Orçamento. Superintendência. Central de Planejamento. Plano Plurianual de Ação Governamental - 2004-2007, volume I. Belo Horizonte: Secretaria de Estado de Planejamento e Gestão; 2004. Disponível em: http://www.planejamento. mg.gov.br/images/documentos/ppag/2004-2007/ppag_ v1_2004_2007.pdf.

24. Minas Gerais. Secretaria de Estado da Saúde de Minas Gerais. Plano Estadual de Saúde: 2008-2011. Belo Horizonte; s/d. Disponível em: http://www.saude.mg.gov.br/images/ documentos/Plano\%20Estadual\%20de\%20Saude.pdf.

25. Secretaria de Estado da Saúde de Minas Gerais. Plano Estadual de Saúde: 2012-2015. Belo Horizonte: Autêntica; 2012. Disponível em: http:/www.saude.mg.gov.br/images/ documentos/Plano\%20estadual\%20de\%20saude_210213 BX.pdf

Recebido em: 03.04.2017

Aceito em: 24.06.2017 